Bull. Austral. Math. Soc.

VoL. 39 (1989) [277-286]

\title{
EPICOMPLETE ARCHIMEDEAN LATTICE-ORDERED GROUPS
}

\author{
DAO-RONG TON
}

In this paper we give the structure of an $\aleph_{i}$-complete $\ell$-group and the epicomplete objects in the category $\mathcal{A}^{l}$.

\section{INTRODUCTION}

We determined the structure of a complete $\ell$-group in [8] and the structure of an Archimedean $\ell$-group in [8]. In this paper we will determine the structue of an $\aleph_{i}$-complete $\ell$-group, in particular, of a $\sigma$-complete $\ell$-group.

Let $\mathcal{L}$ be the category of all $\ell$-homomorphisms between abelian $\ell$-groups. In [1], Anderson and Conrad determined the epicomplete objects in $\mathcal{L}$. An object $G$ in $\mathcal{L}$ is epicomplete if and only if it is divisible. Let $\mathcal{A}^{\ell}$ be the category of all $\ell$-homomorphisms between Archimedean $\ell$-groups. In $[2,3]$, Ball and Hager determined the epicomplete objects in $\mathcal{A}^{\ell}$. An object $G^{\ell}$ in $\mathcal{A}$ is epicomplete if and only if $G$ is divisible and $\sigma$. complete and $\sigma$-laterally complete (meaning each countable subset of positive elements of $G$ which is either bounded or pairwise disjoint has a supremum). In this paper we will give the structure of the epicomplete objects in $\mathcal{A}^{\ell}$.

Our general terminology and notation are standard, as in [5]; for the special notations to be discussed here the reader may refer to $[8,9]$.

\section{The STRUCTURE OF AN $\aleph_{i}$-COMPLETE $\ell$-GROUP}

Let $G$ be an $\ell$-group. We denote the least cardinal $\alpha$ such that $|A| \leqslant \alpha$ for each bounded disjoint subset $A$ of $G$ by $v G$, where $|A|$ denotes the cardinal of $A$. $G$ is said to be $v$-homogeneous if $v H=v G$ for any convex $\ell$-subgroup $H \neq\{0\}$ of the $\ell$-group $G$. Let $G$ be a $v$-homogeneous $\ell$-group and $\aleph_{i}$ a cardinal number. If $v G=\aleph_{i}$, we call $G$ an $\ell$-group of $\aleph_{i}$ type. For example, an $\ell$-group of countable type is an $\ell$-group of $\aleph_{0}$. (For the definition of an $\ell$-group of countable type the reader may consult [10].) The free abelian $\ell$-group $A_{\eta}$ of rank $\eta(\eta>1)$ is an $\ell$-group of $\aleph_{0}$ type (see Proposition 8.1 in [0]). A Riesz space (vector lattice) $V$ is said to be of $\aleph_{i}$ type if it is an $\ell$-group of $\aleph_{i}$ type.

Received 13 May, 1988

The author is indebted to W.C. Holland for his many suggestions and his patience through long hours of discussion of this material.

Copyright Clearance Centre, Inc. Serial-fee code: $0004-9729 / 89 \$ A 2.00+0.00$. 
Lemma 2.1. Any Archimedean $\ell$-group $G$ of $\aleph_{i}$ type with a weak unit can be embedded into a complete Riesz space of $\aleph_{i}$ type.

Proof: Let $G^{\sim}$ be the Dedekind-MacNeille completion of $G$. It follows from Proposition 2.12 in [8] that $G^{-}$is a complete $\ell$-group of $\aleph_{i}$ type. From 1.16 in [6] we see that $G^{\sim}$ can be embedded into a complete Riesz space $U\left(G^{\sim}\right)$; that is

$$
G \rightarrow G^{\sim} \rightarrow Z\left(G^{\wedge}\right) \rightarrow U\left(G^{\wedge}\right)
$$

where $Z\left(G^{-}\right)=\left\{\frac{x}{n} \mid x \in G^{-} \& n \in \mathrm{N}\right\}$ and $U\left(G^{-}\right)$is the Dedekind-MacNeille comple-

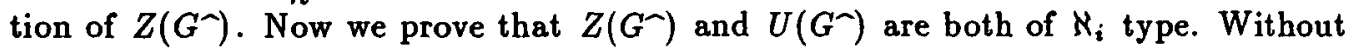
loss of generality, from Proposition 2.2 in [8] we may assume

$$
\sum_{\delta \in \Delta} T_{\delta} \subseteq G^{\sim} \subseteq \prod_{\delta \in \Delta} T_{\delta}
$$

where each $T_{\delta}(\delta \in \Delta)$ is a real group, or an integer group, or a complete $v$ homogeneous $\ell$-group of $\mathcal{N}_{i}$ type. Put $\bar{T}_{\delta}=\left\{g \in G^{-} \mid \delta^{\prime} \neq \delta \Longrightarrow g_{\delta^{\prime}}=0\right\}$ for $\delta \in \Delta$. Then $\bar{T}_{\delta}$ is a convex $\ell$-subgroup of $G^{-}$for $\delta \in \Delta$. So $v \bar{T}_{\delta}=v G^{-}=\aleph_{i}$. Hence each $T_{\delta}(\delta \in \Delta)$ is a continuous complete $\ell$-group of $\aleph_{i}$ type. From (1) we have

$$
\sum_{\delta \in \Delta} T_{\delta} \subseteq G^{\sim} \subseteq Z\left(G^{\wedge}\right) \subseteq \prod_{\delta \in \Delta}^{\prime} Z\left(T_{\delta}\right),
$$

where $Z\left(T_{\delta}\right)=\left\{\frac{x_{\delta}}{n} \mid x_{\delta} \in T_{\delta} \& n \in N\right\}$. Let $\left\{x^{\alpha} \mid \alpha \in A\right\}$ be a disjoint subset with an upper bound in $Z\left(G^{\sim}\right)$. Then there exists a division of $\Delta$ from formula (3)

$$
\Delta=\left(\bigcup_{\alpha \in A} \Delta_{\alpha}\right) \bigcup \Delta^{\prime},
$$

where $\Delta_{\alpha}=\left\{\delta \in \Delta \mid x_{\delta}^{\alpha} \neq 0\right\}$, and $\Delta^{\prime}=\left\{\delta \in \Delta \mid(\forall \alpha \in A)\left(x_{\delta}^{\alpha}=0\right)\right\}$. It is clear that, if $\alpha \neq \alpha^{\prime}$ then $\Delta_{\alpha} \cap \Delta_{\alpha^{\prime}}=\emptyset$. Let $x$ be a weak unit in $G$. Then $x$ is also a weak unit in $G^{-}$. In fact, for any $y \in G^{-}, y=\bigvee_{\alpha \in A}\left\{y_{\alpha} \in G \mid \alpha \in A\right\}$. Then $0=x \wedge y=x \wedge\left(\bigvee_{\alpha \in A} y_{\alpha}\right)=\bigvee_{\alpha \in A}\left(x \wedge y_{\alpha}\right)$ implies $x \wedge y_{\alpha}=0$ for all $\alpha \in A$. So $y=0$. Let $\bar{x}_{\alpha}$ be the element whose $\delta$ component is $x_{\delta}$ and all other components are zero. Put

$$
\overline{x_{\delta}^{\alpha}}= \begin{cases}x_{\delta} & \delta \in \Delta_{\alpha} \\ 0 & \delta \in \Delta_{\alpha},\end{cases}
$$

then $\bar{x}^{\alpha}=\left(\ldots \bar{x}_{\delta}^{\alpha} \ldots\right)=\bigvee_{\delta \in \Delta_{\alpha}}\left(G^{-}\right)_{\bar{x}_{\alpha}}$, hence $\left\{\bar{x}^{\alpha} \mid \alpha \in A\right\}$ is a disjoint subset with an upper bound $x$ in $G^{-}$. So $|A| \leqslant \aleph_{i}$ and $v Z\left(G^{\sim}\right) \leqslant \aleph_{i}$. On the other hand, since 
$G^{\frown} \subseteq Z\left(G^{\frown}\right)$, we have $v Z\left(G^{\wedge}\right) \geqslant v G^{\wedge}=\aleph_{i}$. Therefore, $Z\left(G^{\wedge}\right)$ is of $\aleph_{i}$ type. From Proposition 2.12 in [8] we see that $v \overline{\mathrm{U}}\left(G^{\wedge}\right)=\aleph_{i}$.

A lattice $L$ is called $\aleph_{i}$-complete if each of its non-empty subsets with cardinality number $\alpha \leqslant \aleph_{i}$ has a supremum and an infimum. A Boolean algebra $X$ is said to be $\aleph_{i}$-complete if $X$ is an $\aleph_{i}$-complete lattice. A Boolean algebra $\mathcal{P}$ is called an algebra of $\aleph_{i}$ type if the cardinal number of each disjoint subset is at most $\aleph_{i}$.

Lemma 2.2. Let $\mathcal{P}$ be an $\aleph_{i}$-complete Boolean algebra of $\aleph_{i}$ type. Then in an arbitrary infinite subset $E$ of $\mathcal{P}$ there exists a subset $E^{\prime} \subseteq E$ with $\left|E^{\prime}\right| \leqslant \aleph_{i}$ such that

$$
\vee E^{\prime}=\vee E, \quad \wedge E^{\prime}=\wedge E .
$$

Proof: The proof is similar to the proof of Theorem VI.1.1 in [10]. Let $E$ be an arbitrary infinite subset of $\mathcal{P}$. We denote by $\mathcal{N}$ the set of all subsets $N \subseteq \mathcal{P}$ with $|N| \leqslant \aleph_{i}$ possessing the following properties:

a) $N$ is a disjoint set;

b) if $e \notin N$, then there exists an $e_{1} \in E$ such that $e \leqslant e_{1}$.

The set $\mathcal{N}$ is non-empty. We assume that $\mathcal{N}$ is ordered by inclusion. We will show that $\mathcal{N}$ satisfies the condition of Zorn's Lemma. In fact, if $\mathcal{N}^{\prime} \subset \mathcal{N} \mathcal{N}^{\prime}=\left\{N_{\alpha} \mid \alpha \in A\right\}$ and $\mathcal{N}^{\prime}$ is a chain, then we put $N^{\prime}=\bigcup_{\alpha \in A} N_{\alpha}$. Since for arbitrary $e_{1}, e_{2} \in N^{\prime}$ an index $\alpha$ can be found for which $e_{1}, e_{2} \in N_{\alpha}$, then $N^{\prime}$ consists of pairwise disjoint elements and hence $\left|N^{\prime}\right|$ is at most $N_{i}$ and $N^{\prime} \in \mathcal{N}$. By Zorn's Lemma, there exists a maximal set $N_{0}$ in $\mathcal{N}$. Let $e_{0}=\vee N_{0}$. We can show that $e_{0}=\vee E$. Suppose that there exists $e_{1} \in E$ such that $e_{1}$ is not $\leqslant e_{0}$. Then $e_{1} \wedge e_{0}^{\prime}=e>0$ (see Theorem II.5.2 a), b) in [10]). Now, adjoining the element $e$ to the set $N_{0}$, we obtain a set which also occurs in $\mathcal{N}$ which contradicts the maximality of $N_{0}$. On the other hand, since $N_{0}$ satisfies the condition b), then there exists a subset $E^{\prime}$ in $E$ with $\left|E^{\prime}\right| \leqslant\left|N_{0}\right| \leqslant \aleph_{i}$ such that $e_{0} \leqslant \vee E^{\prime}$. Consequently, $e_{0} \vee E^{\prime}=\vee E$.

A Riesz space $X$ is called a space of $\aleph_{i}$ type if the cardinal number of each bounded disjoint subset is at most $\aleph_{i}$.

Lemma 2.3. Let $X$ be a Dedekind complete Riesz space of $\aleph_{i}$ type. Then in every infinite subset $E \subseteq X$ which is bounded above (below), there exists a subset $E^{\prime} \subseteq E$ with $\left|E^{\prime}\right| \leqslant \aleph_{i}$ such that $\vee E^{\prime}=\vee E\left(\wedge E^{\prime}=\wedge E\right)$.

Proof: This is similar to the proof of Theorem VI.2.2 in [10], using Lemma 2.2 to replace Theorem VI.1.1.

Lemma 2.4. In any Archimedean $\ell$-group $G$ of $\aleph_{i}$ type with a weak unit, if $z=\bigvee_{\alpha \in A}(G)_{z_{\alpha}}$, then there exists a subset $\left\{z_{\alpha^{\prime}} \mid \alpha^{\prime} \in A\right\}$ with $\left|A^{\prime}\right| \leqslant \aleph_{i}$ of $\left\{z_{\alpha} \mid \alpha \in A\right\}$ 
such that $z=\bigvee_{\alpha^{\prime} \in A^{\prime}}^{(G)} z_{\alpha^{\prime}}$

Proof: Let $G$ be an Archimedean $\ell$-group of $\aleph_{i}$ type with a weak unit. From Lemma 2.1 we see $G$ can be embedded into a complete Riesz space $U(G)$ of $\aleph_{i}$ type according to the process of (1). Since $U(G)$ is a regular extension of $Z(G)$, we can assume

$$
z^{\prime}=\bigvee_{\alpha \in A}^{(Z(G))} z_{\alpha}=\bigvee_{\alpha \in A}^{(U(G))} z_{\alpha}
$$

By Lemma 2.3 there exists a subset $\left\{z_{\alpha^{\prime}} \mid \alpha^{\prime} \in A^{\prime}\right\}$ with $\left|A^{\prime}\right| \leqslant \aleph_{i}$ of $\left\{z_{\alpha} \mid \alpha \in A\right\}$ such that

$$
z^{\prime}=\bigvee_{\alpha^{\prime} \in A^{\prime}}^{(Z(G))} z_{\alpha^{\prime}}=\bigvee_{\alpha^{\prime} \in A^{\prime}}^{(U(G))} z_{\alpha^{\prime}}
$$

We denote the set of all upper bounds of the subset $M$ of $G$ in $Z(G)$ by $M_{Z(G)}^{\star}$ and the set of all upper bounds of $M$ in $G$ by $M_{G}^{\star}$. From (4) and (5) we have

$$
\left\{z_{\alpha} \mid \alpha \in A\right\}_{Z(G)}^{\star}=\left\{z_{\alpha^{\prime}} \mid \alpha^{\prime} \in A^{\prime}\right\}_{Z(G)}^{\star}
$$

then

$$
\begin{aligned}
\left\{z_{\alpha} \mid \alpha \in A\right\}_{Z(G)}^{\star} \cap G & =\left\{z_{\alpha^{\prime}} \mid \alpha^{\prime} \in A^{\prime}\right\}_{Z(G)}^{\star} \cap G \\
\left\{z_{\alpha} \mid \alpha \in A\right\}_{G}^{\star} & =\left\{z_{\alpha^{\prime}} \mid \alpha^{\prime} \in A\right\}_{G}^{\star}
\end{aligned}
$$

Therefore

$$
\bigvee_{\alpha^{\prime} \in A^{\prime}}^{(G)} z_{\alpha^{\prime}}=\bigvee_{\alpha \in A}^{(G)} z_{\alpha}=z
$$

An $\ell$-group $G$ is said to be $\aleph_{i}$-complete if each upper bounded subset $E$ with $|E| \leqslant \aleph_{i}$ in $G$ has a least upper bound. For example, a $\sigma$-complete $\ell$-group $G$ is $\aleph_{0}$-complete. Since a $\sigma$-complete $\ell$-group is Archimedean, if $\aleph_{i} \geqslant \aleph_{0}$, an $\aleph_{i}$-complete $\ell$-group is Archimedean.

LEMMA 2.5. Any $\aleph_{i}$-complete $\ell$-group of $\aleph_{i}$ type with a weak unit is complete.

Proof: Let $G$ be an $\aleph_{i}$-complete $\ell$-group of $\aleph_{i}$ type with a weak unit $\varepsilon$. Then $G$ is an Archimedean $\ell$-group of $\aleph_{i}$ type with a weak unit $\varepsilon$ and $G$ has a Dedekind completion $G^{-}$. Let $\left\{x^{\alpha} \mid \alpha \in A\right\}$ be an arbitrary upper bounded subset in $G$. Assume

$$
x=\bigvee_{\alpha \in A}\left(\hat{G}^{\wedge}\right)_{x^{\alpha}} .
$$


By Proposition 2.12 in [8] we see that $G^{\sim}$ is also an $\ell$-group of $\aleph_{i}$ type. And $\varepsilon$ is also a weak unit in $G^{-}$. So, by Lemma 2.4 , there exists a subset $\left\{x^{\alpha^{\prime}} \mid \alpha^{\prime} \in A^{\prime}\right\}$ with $\left|A^{\prime}\right| \leqslant \aleph_{i}$ of $\left\{x^{\alpha} \mid \alpha \in A\right\}$ such that

$$
x=\bigvee_{\alpha^{\prime} \in A^{\prime}}\left(G^{\wedge}\right)_{x^{\alpha^{\prime}}} .
$$

Therefore

$$
x=\bigvee_{\alpha^{\prime} \in A^{\prime}}^{(G)} x^{\alpha^{\prime}}
$$

Lemma 2.6. Let $G$ be an Archimedean $\ell$-group. If the completion $G^{-}$has a weak unit, then $G$ also has a weak unit.

Proof: Let $\varepsilon$ be a weak unit in $G^{-}$. From Theorem 2.4 in [4] there exists $\varepsilon^{\prime}$ in $G$ such that $\varepsilon^{\prime} \geqslant \varepsilon$. Then $\varepsilon^{\prime}$ is a weak unit in $G$. Because, if $\varepsilon^{\prime} \wedge x=0$ for $x \in G$, then $\varepsilon \wedge x=0$. Hence $x=0$.

Let $\ell$-group $G$ be a subdirect sum of $\left\{G_{\delta} \mid \delta \in \Delta\right\}$. If there exists a subset $\Delta_{1} \subseteq \Delta$ such that $\sum_{\delta \in \Delta_{1}} G_{\delta} \subseteq G$, then we call $G$ a semicomplete subdirect sum of $\left\{G_{\delta} \mid \delta \in \Delta\right\}$.

An $\ell$-group $G$ is said to be projectable or a $P$-group, if $G=g^{\perp \perp} \boxplus g^{\perp}$ for each $g \in G$, where $g^{\perp}=\{g\}^{\perp}=\{x \in G|| g|\wedge| x \mid=0\}$ and $g^{\perp \perp}=\left(g^{\perp}\right)^{\perp}$. It is well-known that any $\sigma$-complete $\ell$-group $G$ is projectable. So any $\aleph_{i}$-complete $\ell$-group $G$ is projectable for $\aleph_{i} \geqslant \aleph_{0}$. An Archimedean $\ell$-group $G$ is said to be continuous, if for any strictly positive element $x$ we have $x=x_{1}+x_{2}$ and $x_{1} \wedge x_{2}=0$, where $x_{1} \neq 0$ and $x_{2} \neq 0$.

Lemma 2.7. Any $\aleph_{i}$-complete $\ell$-group $G$ of $\aleph_{j}$ type with $\aleph_{i}$ and $\aleph_{j} \geqslant \aleph_{0}$ is continuous.

Proof: Let $0<x \in G$. Since $v[x]=v G=\aleph_{j}$, by 4.3 in [7], $[0, x]$ is not a chain, and there exists $0<x_{1}<x$ and $0<x_{2}<x$ such that $x_{2} \wedge x_{1}=0$. It is clear that $x^{\perp \perp}$ is also $\aleph_{i}$-complete. In fact, if $\left\{x^{\alpha} \mid \alpha \in A\right\}$ is a subset in $x^{\perp \perp}$ with $|A| \leqslant \aleph_{i}$ and $x^{\alpha} \leqslant \bar{x} \in x^{\perp \perp}$ for $\alpha \in A$, then there exists $\bigvee^{(G)} x^{\alpha}=x_{0} \in G$. Since $\bar{x} \wedge y=0$ for each $y \in x^{\perp}, x_{0} \wedge y=0$ for each $y \in x^{\perp}$ and so $x_{0} \in x^{\perp \perp}$. Because $G$ is projectable, we have

$$
x^{\perp \perp}=x_{1}^{\perp \perp} \boxplus x_{1}^{\perp} \text {. }
$$

It is easy to see that $x \bar{\epsilon} x_{1}^{\perp \perp}$. In fact, if $x \in x_{1}^{\perp \perp}$, then $x^{\perp \perp} \subseteq x_{1}^{\perp \perp}$, hence $x^{\perp \perp}=x_{1}^{\perp \perp}$. But $x_{2} \in x_{1}^{\perp} \subseteq x^{\perp \perp}$, giving a contradiction. On the other hand, since $x_{1} \bar{\epsilon} x_{1}^{\perp}$, we have 
$x \bar{\epsilon} x_{1}^{\perp}$. From (6) we see that there exist $0<x^{1} \in x_{1}^{\perp \perp}$ and $0<x^{2} \in x_{1}^{\perp}$ such that $x=x^{1}+x^{2}$. Therefore $G$ is continuous.

THEOREM 2.8. Any $\aleph_{i}$-complete $\ell$-group $G$ is $\ell$-isomorphic to an semicomplete subdirect sum of real groups, integer groups, continuous complete $\ell$-groups of $\aleph_{i}$ type and continuous $\aleph_{i}$-complete $\ell$-groups of $\aleph_{j}$ type with $\aleph_{j}>\aleph_{i}$.

ProOF: We will proceed in three steps.

(1) Let $G$ be an $\aleph_{i}$-complete $\ell$-group. Then $G$ has a Dedekind completion $G^{-}$. From Theorem III.4.4 and Theorem III.4.6 in [11] we see that

$$
G^{\sim} \subseteq \prod_{\lambda \in \Lambda} G_{\lambda},
$$

where $G_{\lambda}$ is a complete $\ell$-group with a weak unit $x_{\lambda}$ for $\lambda \in \Lambda$. From Proposition 2.2 in [8], without loss of generality, we have

$$
G_{\lambda} \subseteq{ }^{\star} \prod_{\lambda_{\delta} \in \Delta_{\lambda}} G_{\lambda_{\delta}}
$$

for each $\lambda \in \Lambda$, where $G_{\lambda_{\delta}}\left(\lambda_{\delta} \in \Delta_{\lambda}\right)$ is a real group, or an integer group or a continuous $v$-homogeneous complete $\ell$-group. We can show that $x_{\lambda_{6}}$ is a weak unit in $G_{\lambda_{\delta}}$ for each $\lambda_{\delta} \in \Delta_{\lambda}$. In fact, let $y_{\lambda_{\delta}} \in G_{\lambda_{\delta}}$ and $x_{\lambda_{\delta}} \wedge y_{\lambda_{\delta}}=0$. Let $\bar{y}_{\lambda_{\delta}}$ be the element in $G_{\lambda_{\delta}}$ whose $\lambda_{\delta}$ component is $y_{\lambda_{\delta}}$ and all other components are zero. Then $x_{\lambda} \wedge \bar{y}_{\lambda_{\delta}}=0$ and so $\bar{y}_{\lambda_{\delta}}=0$, therefore $y_{\lambda_{\delta}}=0$.

From (7) and (8) we have

$$
G^{\sim} \subseteq^{*} \prod_{\lambda \in \Lambda} G_{\lambda} \subseteq{ }^{*} \prod_{\lambda \in \Lambda}\left(\prod_{\lambda_{\delta} \in \Delta_{\lambda}} G_{\lambda_{\delta}}\right) \subseteq^{*} \prod_{\substack{\lambda \in \Lambda \\ \lambda_{\delta} \in \Delta_{\lambda}}} G_{\lambda_{\delta}}
$$

Putting $\Delta=\bigcup_{\lambda \in \Lambda} \Delta_{\lambda}$ and $T_{\delta}=G_{\lambda_{\delta}}$ for each $\delta \in \Delta$, we get

$$
G^{-} \underline{\complement}^{\star} \prod_{\delta \in \Delta} T_{\delta}
$$

Let $\rho_{\delta}$ be the projection from $\prod_{\delta \in \Delta} T_{\delta}$ to $T_{\delta}$ and $T_{\delta}^{\prime}=G \rho_{\delta}$ for each $\delta \in \Delta$. Then

$$
G \subseteq^{\prime} \prod_{\delta \in \Delta} T_{\delta}^{\prime}
$$

where each $T_{\delta}$ is a real group, or an integer group or a continuous $v$-homogenous complete $\ell$-group with a weak unit and $T_{\delta}^{\prime}$ is a subgroup of reals or a $v$-homogenous Archimedean $\ell$-group with a weak unit for $\delta \in \Delta$ (see Lemma 2.6). 
(2) It is clear that the projection $\rho_{\delta}$ from $G$ onto $T_{\delta}^{\prime}$ is complete. Let $\left\{x_{\delta}^{\alpha} \mid \alpha \in A\right\}$ be a subset in $T_{\delta}^{\prime}$ with $|A| \leqslant \aleph_{i}$ and $x_{\delta}^{\alpha} \leqslant x_{\delta} \in T_{\delta}^{\prime}$ for $\alpha \in A$. Let $x^{\alpha}$ be the element in $G$ whose $\delta$ component is $x_{\delta}^{\alpha}$ and $x$ the element in $G$ whose $\delta$ component is $x_{\delta}$. Put $y^{\alpha}=x^{\alpha} \wedge x$. Then $\left\{y^{\alpha} \mid \alpha \in A\right\}$ is a subset of $G$ with an upper bound $x$ and $|A| \leqslant \aleph_{i}$. Thus there exists $y=\bigvee_{\alpha \in A}{ }^{(G)} y^{\alpha}$. Clearly, $y^{\alpha} \rho_{\delta}=x_{\delta}^{\alpha}$. Put $y \rho_{\delta}=y_{\delta} \in T_{\delta}^{\prime}$. Then $y_{\delta}=\bigvee_{\alpha \in A}\left(T_{\delta}^{\prime}\right)_{x_{\delta}^{\alpha}}$. So each $T_{\delta}^{\prime}$ is $\aleph_{i}$-complete for $\delta \in \Delta$. If $T_{\delta}^{\prime}$ is a subgroup of the reals, then $T_{\delta}^{\prime}$ is $\mathbf{R}$ or $Z$. If $T_{\delta}^{\prime}$ is not a subgroup of the reals, then $T_{\delta}^{\prime}$ is $v$-homogeneous. Suppose $v T_{\delta}^{\prime}=\aleph_{j}$. If $\aleph_{j}=\aleph_{i}, T_{\delta}^{\prime}$ is an $\aleph_{i}$-complete $\ell$-group of $\aleph_{i}$ type. It follows from Lemma 5 that $T_{\delta}^{\prime}$ is complete for those $\delta \in \Delta$ for which $T_{\delta}^{\prime}$ is of $\aleph_{i}$ type. It then follows from Lemma 2.7 that each $T_{\delta}^{\prime}$ is continuous for $\delta \in \Delta$.

(3) Finally we prove $G$ is a semicomplete subdirect sum. Put $\Delta_{1}=\left\{\delta \in \Delta \mid T_{\delta}^{\prime}\right.$ is $\mathbf{R}$ or $\mathbf{Z}$ or a continuous complete $\ell$-group of $\aleph_{i}$ type $\}$. For each $\delta \in \Delta_{1}$, set

$$
\bar{T}_{\delta}^{\prime}=\left\{g \in G \mid \delta^{\prime} \neq \delta \Longrightarrow g_{\delta^{\prime}}=0\right\} .
$$

Let $\bar{z}_{\delta}$ be the strictly positive element in $\bar{T}_{\delta}^{\prime}$ whose $\delta$ component is $z_{\delta}$. Then $\bar{z}_{\delta} \in G^{-}$ because $G^{\wedge} \supseteq \sum_{\delta \in \Delta} T_{\delta} \supseteq \sum_{\delta \in \Delta} T_{\delta}^{\prime}$. From Theorem 1.1 in [4] we have

$$
\bar{z}_{\delta}=\bigvee_{\alpha \in A}^{\hat{G}^{\hat{N}}}\left\{z^{\alpha} \in G \mid 0 \leqslant z^{\alpha} \leqslant \bar{z}_{\sigma}\right\}
$$

It is clear that $z^{\alpha} \in=\bar{T}_{\delta}^{\prime}$. It follows from (10) that

$$
\bar{z}_{\sigma}=\bigvee_{\alpha \in A}\left(T_{\sigma}^{\prime}\right)_{z_{\delta}^{\alpha}}
$$

By Lemma 2.4 there exists a subset $\left\{z^{\alpha^{\prime}} \mid \alpha^{\prime} \in A^{\prime}\right\}$ with $\left|A^{\prime}\right| \leqslant \aleph_{i}$ of $\left\{z^{\alpha} \mid \alpha \in A\right\}$ such that

$$
z_{\delta}=\bigvee_{\delta^{\prime} \in A^{\prime}}\left(T_{\delta}^{\prime}\right) z^{\alpha^{\prime}}
$$

Therefore

$$
\bar{z}_{\delta}=\bigvee_{\alpha^{\prime} \in A^{\prime}}\left(G^{\prime}\right) z_{\delta}^{\alpha^{\prime}}
$$

From Theorem 2.4 in [4] there exists $z^{\prime} \in G$ such that $\bar{z}_{\delta} \leqslant z^{\prime}$. Since $G$ is $\aleph_{i}$-complete, there exists $\bigvee_{\alpha^{\prime} \in A^{\prime}}{ }^{(G)} z^{\alpha^{\prime}}$. From Lemma 2.2 and Theorem 2.4 in [4] and (11) above, we see that

$$
\bigvee_{\alpha^{\prime} \in A^{\prime}}^{(G)} z^{\alpha^{\prime}}=\bigvee_{\alpha^{\prime} \in A^{\prime}}\left(G^{\wedge}\right) z^{\alpha^{\prime}}=\bar{z}_{\alpha}
$$


Thus $\bar{z}_{\delta} \in G$. This proves that $\bar{T}_{\delta}^{\prime} \subseteq G$ for each $\delta \in \Delta_{1}$. Therefore

$$
\sum_{\delta \in \Delta_{1}} T_{\delta}^{\prime} \subseteq G \subseteq^{\prime} \prod_{\delta \in \Delta} T_{\delta}^{\prime}
$$

\section{EPICOMPLETE OBJECTS IN THE CATEgORY $\mathcal{A}^{\ell}$}

Lemma 3.1. The following are equivalent for $G \in \mathcal{A}$ :

(a) $G$ is epicomplete in $\mathcal{A}^{\ell}$;

(b) $G$ is conditionally and laterally $\sigma$-complete, and $G$ is divisible (see Theorem 4.9 in [3]).

From Theorem 2.8 we have the following result.

COROLlary 3.2. Any $\sigma$-complete $\ell$-group is $\ell$-isomorphic to a semicomplete subdirect sum of real groups, integer groups, continuous complete $\ell$-groups of countable type and continuous $\sigma$-complete $\ell$-groups of $\aleph_{j}$ type with $\aleph_{j}>\aleph_{0}$. That is, there exists an $\ell$-isomorphism $f$ such that

$$
\sum_{\delta \in \Delta_{1} \subseteq \Delta} T_{\delta} \subseteq f(G) \subseteq^{\prime} \prod_{\delta \in \Delta} T_{\delta}
$$

where $\Delta_{1}=\left\{\delta \in \Delta \mid T_{\delta}\right.$ is a real group, or an integer group or a continuous complete $\ell$-group of countable type $\}$ and $\Delta \backslash \Delta_{1}=\left\{\delta \in \Delta \mid T_{\delta}\right.$ is a continuous $\sigma$-complete $\ell$ group of $\aleph_{j}$ type with $\left.\aleph_{j}>\aleph_{0}\right\}$.

Now let $G$ be a divisible $\sigma$-complete $\ell$-group. Without loss of generality, from (12) we have

$$
\sum_{\delta \in \Delta_{1} \subseteq \Delta} T_{\delta} \subseteq G \subseteq \prod_{\delta \in \Delta} T_{\delta} .
$$

Each $T_{\delta}$ is a homomorphic image of $G$, hence $T_{\delta}$ is divisible for each $\delta \in \Delta$. Thus we get:

Corollary 3.3. Any divisible $\sigma$-complete $\ell$-group is $\ell$-isomorphic to a semicomplete subdirect sum of real groups, continuous divisible complete $\ell$-groups of ocuntable type and continuous divisible $\sigma$-complete $\ell$-groups of $\aleph_{j}$ type with $\aleph_{j}>\aleph_{0}$.

Let $\left\{T_{\delta} \mid \delta \in \Delta\right\}$ be a set of $\ell$-groups. Put

$$
\prod_{\delta \in \Delta}{ }^{\sigma} T_{\delta}=\left\{x \in \prod_{\delta \in \Delta} T_{\delta} \mid \exists \text { a countable subset } \Delta_{\sigma} \text { in } \Delta \text { such that } x_{\delta}=0 \text { if } \delta \bar{\epsilon} \Delta_{\sigma}\right\} \text {. }
$$

Let $\ell$-group $G$ be a semicomplete subdirect sum of $\left\{T_{\delta} \mid \delta \in \Delta\right\}$. That is.

$$
\sum_{\delta \in \Delta_{1} \subseteq \Delta} T_{\delta} \subset G \subseteq^{\prime} \prod_{\delta \in \Delta} T_{\delta} .
$$

If $\prod_{\delta \in \Delta_{1}}{ }^{\sigma} T_{\delta} \subseteq G$, then we call $G$ a $\sigma$-semicomplete subdirect sum of $\left\{T_{\delta} \mid \delta \in \Delta\right\}$. 
Theorem 3.4. Let $G$ be an epicomplete object in the category $\mathcal{A}^{\ell}$. Then $G$ is $\ell$-isomorphic to a $\sigma$-semicomplete subdirect sum of real groups, continuous complete epicomplete $\ell$-groups of countable typs and continuous $\ell$-groups of $\aleph_{j}$ type with $\aleph_{j}>$ $\aleph_{0}$.

Proof: By Lemma 3.1 and Corollary 3.3 we have

$$
\sum_{\delta \in \Delta_{1} \subseteq \Delta} T_{\delta} \subseteq G \subseteq \prod^{\prime} \prod_{\delta \in \Delta} T_{\delta}
$$

where $T_{\delta}$ is a real group or a continuous divisible complete $\ell$-group of countable type for each $\delta \in \Delta_{1}$ and $T_{\delta}$ is a continuous divisible $\sigma$-complete $\ell$-group of $\aleph_{j}$ type with $\aleph_{j}>\aleph_{0}$. By 6.1 in [2] the real group $R$ is epicomplete in $\mathcal{A}^{l}$. For each $\delta \in \Delta_{1}$ put

$$
\bar{T}_{\delta}=\left\{g \in G \mid \delta^{\prime} \neq \delta \Longrightarrow g_{\delta}=0\right\}
$$

From (13) we have

$$
G=\bar{T}_{\delta} \boxplus G_{\delta}
$$

for each $\delta \in \Delta_{1}$, where $G_{\delta}=\left\{g \in G \mid g_{\delta}=0\right\}$. If $\bar{T}_{\delta} \leqslant T_{\delta}^{\prime}$ is epic in $\mathcal{A}^{\ell}$, put $G^{\prime}=T_{\delta}^{\prime} \boxplus G_{\delta}$. Then $G \leqslant G^{\prime}$. Suppose $\alpha_{1}$ and $\alpha_{2}$ are two $\ell$-homomorphisms from $G^{\prime}$ to an Archimedean $\ell$-group $P$ such that $\left.\alpha_{1}\right|_{G}=\left.\alpha_{2}\right|_{G}$. Then $\left.\alpha_{1}\right|_{\bar{T}_{\delta}}=\left.\alpha_{2}\right|_{\bar{T}_{\delta}}$. So $\left.\alpha_{1}\right|_{T_{\delta}^{\prime}}=\left.\alpha_{2}\right|_{T_{\delta}^{\prime}}$ and $\alpha_{1}=\alpha_{2}$. This means $G \leqslant G^{\prime}$ is epic in $\mathcal{A}^{l}$. Since $G$ is epicomplete, $G=G^{\prime}$ and $\bar{T}_{\delta}=T_{\delta}^{\prime}$. Therefore each $\bar{T}_{\delta}$ or $T_{\delta}$ is epicomplete in $\mathcal{A}^{\ell}$ for $\delta \in \Delta_{1}$.

On the other hand, $G$ is $\sigma$-laterally complete. Hence

$$
\prod_{\delta \in \Delta_{1}}{ }^{\sigma} T_{\delta} \subseteq G \subseteq \prod_{\delta \in \Delta} T_{\delta}
$$

\section{REFERENCES}

[1] M. Anderson and P.F. Conrad, 'Epicomplete l-groups', Algebra Universalis 12 (1981), $224-241$.

[2] R.N. Ball and A.W. Hager, 'Epimorphisms in archimedean $\ell$-groups and vector lattices' (to appear), (Bowling Green text on $\ell$-groups).

[3] R.N. Ball and A.W. Hager, 'Epicomplete archimedean $\ell$-groups', (submitted).

[4] P. Conrad and D. Mcalister, 'The completion of a lattice ordered group', J. Austral. Math. Soc. $\theta$ (1969), 182-208.

[5] P. Conrad, Lattice Ordered Groups, Lecture Notes (Tulane University, 1970 ).

[6] Ј. Jakubik, 'Представление И Расширение ८-Групп', Czech. Math. J. 13 (1963), 267-283.

[7] J. Jakubik, 'Homogeneous lattice ordered groups', Czech. Math. J. 22 (1972), 325-337.

[8] Dao-Rong Ton, 'The structure of a complete $\ell$-group'. (submitted) .

[0] Dao-Rong Ton, 'Some properties of an Archimedean $\ell$-group'. (submitted) . 
[10] B.Z. Vulikh, Introduction to the theory of partially ordered spaces (Wolter-Noordhoff, Ltd, Groningen, 1967).

[11] Zheng-pan Yan, Introduction to Partially Ordered Spaces, (in Chinese) (Scientific Publishing House of China, 1964).

Department of Mathematics and Physics, Hohia University,

Jingsu Province,

Nanjing,

People's Republic of China.
Department of Mathematics and Statistics, Bowling Green State University, Bowling Green, Oh. 43403-0221

United States of America. 